\title{
Análise da produção científica fonoaudiológica brasileira sobre paralisia cerebral
}

\author{
Analysis of the Brazilian Speech-Language Pathology scientific \\ production regarding cerebral palsy
}

\author{
Marisa Cláudia Feital da Silva ${ }^{1}$,Sílvia Friedman²
}

\begin{abstract}
RESUMO
Objetivo: Analisar a produção científica fonoaudiológica brasileira sobre paralisia cerebral entre os anos de 2002 a 2009, publicados em periódicos em Fonoaudiologia e em periódicos de áreas afins. Métodos: Estudo documental descritivo pautado na seleção e análise de artigos científicos sobre paralisia cerebral quanto: ao período da publicação; à distribuição de frequência por período; ao procedimento metodológico empregado; à vertente epistemológica adotada; à faixa etária dos sujeitos estudados; à área de atuação fonoaudiológica a que pertence o estudo. Resultados: Foram encontrados 36 artigos: 28 em periódicos em Fonoaudiologia e oito em periódicos de áreas afins. O maior número de publicações concentrou-se entre 2002 e 2005. Quanto à área, a maioria envolve linguagem e motricidade orofacial. Quanto à vertente epistemológica, a maioria era positivista. Os procedimentos metodológicos mais encontrados foram o levantamento e o estudo de caso. A faixa etária mais estudada foi a da infância. Conclusão: O crescimento da produção científica fonoaudiológica brasileira sobre paralisia cerebral não acompanha a trajetória de crescimento em outras áreas da Fonoaudiologia; já a sua distribuição pelas vertentes epistemológicas, sim. Os dados mostraram estudos ligados à audição, à saúde coletiva e à voz, bem como estudos de natureza documental e estudos sobre adolescentes e adultos.
\end{abstract}

Descritores: Paralisia cerebral; Indicadores de produção científica; Produção científica e técnica; Bibliografia como assunto; Revisão

\section{INTRODUÇÃO}

A Fonoaudiologia brasileira completou 27 anos de reconhecimento legal (Lei 6965/81) e alguns autores ${ }^{(1,2)}$ têm apontado o amadurecimento da profissão pelo aumento quantitativo e qualitativo da produção científica em cursos de pós-graduação stricto sensu e em artigos publicados em periódicos fonoaudiológicos e de outras áreas afins.

Esse amadurecimento se mostra, também, pelo aumento do indicador de impacto $^{(2)}$ na produção científica fonoaudiológica, indicador esse que se refere ao número de vezes em que os artigos publicados em periódicos são referenciados em publicações subsequentes.

Sobre a importância dos periódicos, os autores comentam que: (...) estudantes de graduação e de pós-graduação (...) dependem dos periódicos científicos (...) e há uma respeitável

Trabalho realizado na Pontifícia Universidade Católica de São Paulo - PUCSP - São Paulo (SP), Brasil.

(1) Mestre, Fonoaudióloga clínica, São Paulo (SP), Brasil.

(2) Doutora, Professora do Curso de Fonoaudiologia da Pontifícia Universidade Católica de São Paulo - PUC-SP - São Paulo (SP), Brasil.

Endereço para correspondência: Marisa Cláudia Feital da Silva. R. Manuel Álvares da Costa, 187/71B, Jd. Ester Yolanda, São Paulo (SP), Brasil, CEP: 05374-100.E-mail: marisafeital@uol.com.br

Recebido em: 9/12/2009; Aceito em: 24/6/2010 população (de leitores) que depende de periódicos para obter as primeiras informações sobre descobertas importantes ${ }^{(3)}$.

Comentam ainda que o periódico é uma fonte importante de informação e atualização profissional, principalmente, se for considerado que cinco sextos de conhecimento novo em uma dada área serão criados após a graduação de cada profissional e a metade dos artigos que são publicados dizem respeito a assuntos inéditos em sua área de atuação. Referem, também, que não se pode esquecer que o uso de meios eletrônicos aumentou a procura por periódicos, em função da facilidade de utilização e maior variedade de fontes a consultar ${ }^{(3)}$.

A função principal do periódico é o registro e a difusão dos conhecimentos científicos existentes, favorecendo a comunicação entre os pesquisadores e a comunidade cientifica. O periódico científico contribui para o desenvolvimento, a atualização e os avanços científicos, cumprindo funções que permitem a ascensão do cientista para efeitos de promoção, reconhecimento e conquista de poder em seu meio ${ }^{(3-6)}$.

Vários autores têm se dedicado a avaliar a produção em periódicos científicos especializados, tendo em vista que estes são veículos de disseminação, acessibilidade e visibilidade da produção do conhecimento. Seu prestígio acadêmico está ligado à definição, aplicação e divulgação de critérios de qualidade que devem nortear tal produção ${ }^{(4,5,7,8)}$.

Nesse contexto, no presente artigo o foco de estudo são as 
publicações sobre a paralisia cerebral (PC), afecção crônica que pode acometer a pessoa desde antes de seu nascimento até a infância. Atinge o Sistema Nervoso Central, causando problemas no tônus muscular, na postura e na coordenação e realização de movimentos ${ }^{(9)}$. Tais alterações podem comprometer a linguagem, a audição, a voz e a fala: aspectos com os quais trabalha o fonoaudiólogo.

Estudos desta natureza são importantes, porque um panorama da produção científica em geral fornece não apenas uma visão da direção do avanço das pesquisas sobre um dado assunto, como também permite apontar as lacunas existentes e revelar novos aspectos a serem investigados ou aprofundados, bem como a presença de diferentes visões sobre um mesmo tema, estimulando novas pesquisas ${ }^{(2,7,10,11)}$.

O objetivo do presente artigo é estudar a produção científica Fonoaudiologia brasileira sobre paralisia cerebral no período que vai do ano de 2002 ao primeiro semestre do ano de 2009, quanto: ao período da publicação; à distribuição de frequência por período; ao procedimento metodológico empregado; à vertente epistemológica adotada; à faixa etária dos sujeitos estudados; à área de atuação fonoaudiológica envolvida no estudo.

\section{MÉTODOS}

Trata-se de estudo documental descritivo, no qual se analisaram as publicações sobre paralisia cerebral (PC) feitas por fonoaudiólogos, em periódicos de Fonoaudiologia e em periódicos de áreas afins, no período de 2002 ao primeiro semestre de 2009.

Quanto aos periódicos, foram levantados os que possuíssem o ISSN (International Standard Serial Number) e considerados os artigos que tivessem pelo menos um autor fonoaudiólogo. Quanto aos artigos publicados em periódicos científicos brasileiros de Fonoaudiologia, a pesquisa foi feita pela internet, nos sites das revistas e por meio de consulta aos exemplares impressos quando essas informações não estivessem disponíveis na forma online. Foram analisadas as revistas de Fonoaudiologia: Pró-Fono Revista de Atualização Científica; Revista Cefac Atualização Científica em Fonoaudiologia; Revista da Sociedade Brasileira de Fonoaudiologia; Revista Distúrbios da Comunicação. Quanto aos artigos publicados por fonoaudiólogos em periódicos científicos brasileiros de especialidades afins, a consulta foi feita às bases de dados LILACS e MEDLINE por meio do formulário avançado, utilizando-se, no item "palavras", o termo paralisia cerebral; no item "idioma", o Português e no item "país", Brasil. Quanto aos procedimentos metodológicos, os artigos foram classificados em: estudo documental, estudo de caso, levantamento e experimento ${ }^{(12)}$.

\section{Procedimento}

A análise das publicações foi organizada a partir da distribuição de sua frequência, levando-se em conta o período das publicações, o tipo de procedimento metodológico empregado, a vertente epistemológica adotada, a faixa etária dos sujeitos estudados nas publicações e a área da Fonoaudiologia envolvida no estudo.
Para compreender as características teórico-conceituais que subsidiam as publicações e caracterizar sua vertente epistemológica, os trabalhos foram lidos na íntegra.

As temáticas geralmente já se evidenciavam no próprio título do trabalho. A leitura integral do texto ajudou a corroborar a pertinência da definição da temática. Essa definição obedeceu ao critério da abrangência, de modo a permitir que se congregasse o maior número possível de trabalhos em cada uma.

Quanto à área de atuação fonoaudiológica, com base no Conselho Regional de Fonoaudiologia consideraram-se as seguintes: Linguagem, Audiologia, Motricidade Orofacial, Voz e Saúde Coletiva ${ }^{(13)}$.

Quanto à vertente epistemológica, com base nos estudos de filosofia da ciência ${ }^{(14)}$, consideraram-se a positivista, que se refere à visão centrada no organismo e nos sintomas de fala; a dialético-histórica, que se refere à visão centrada no sujeito, sua subjetividade e sua história e a fenomenológica, que se refere a aquilo que o pesquisador pode apreender do objeto, estudando-o a partir de sua percepção.

Quanto à faixa etária, consideraram-se como crianças, sujeitos de até dez anos e 11 meses de idade; adolescentes, sujeitos de 11 a 18 anos de idade e adultos, sujeitos acima dos 18 anos de idade ${ }^{(15)}$.

Quanto ao procedimento metodológico empregado, três tipos de procedimento foram encontrados: o levantamento, em que se mostram avaliações de pacientes com características semelhantes escolhidos aleatoriamente; estudo de caso, em que se mostram investigações aprofundadas de doença ou a intervenção em um único paciente e o experimento, em que se mostrou a influência entre variáveis em grupos de indivíduos com uma característica clínica de interesse (doentes), em comparação com grupos de pessoas que não a possuem ${ }^{(12)}$.

Para a análise estatística dos dados utilizou-se o teste Qui-quadrado $\left(\mathrm{X}^{2}\right)$, tomando 5\% como nível de significância. Os resultados obtidos $\left(\mathrm{X}^{2}\right.$, graus de liberdade e p-valor) são apresentados ao final das tabelas.

\section{RESULTADOS}

De 2002 a 2009 foram encontrados 36 artigos sobre PC, estando 28 em periódicos de Fonoaudiologia e oito em periódicos de áreas afins.

As revistas científicas de áreas afins que publicaram artigos sobre PC com pelo menos um autor fonoaudiólogo, no período de 2002 a 2009, foram: Revista Brasileira de Otorrinolaringologia; Revista Brasileira de Neuro-psiquiatria; Salusvita; Revista Brasileira de Educação Especial e Arquivo Brasileiro de Paralisia Cerebral que corresponderam, respectivamente, às áreas de: Otorrinolaringologia; Neuropsiquiatria; Fisioterapia e Terapia Ocupacional; Educação Especial; Multidisciplinaridade.

A Tabela 1 mostra a distribuição da produção científica brasileira sobre PC no período que vai do ano de 2002 ao primeiro semestre do ano de 2009, de acordo com o periódico em que foram publicados.

A Tabela 2 mostra a distribuição dos artigos pelas áreas de atuação fonoaudiológicas e a Tabela 3 a distribuição da produção das áreas de atuação pelas vertentes epistemológi- 
Tabela 1. Distribuição, por periódicos, da produção científica sobre paralisia cerebral, no período de 2002 a 2008

\begin{tabular}{|c|c|c|c|c|c|c|c|c|c|c|c|c|}
\hline \multirow[t]{2}{*}{ Ano } & \multicolumn{2}{|c|}{ CEFAC } & \multicolumn{2}{|c|}{ Outras } & \multicolumn{2}{|c|}{ Pro-fono } & \multicolumn{2}{|c|}{ SBFa } & \multicolumn{2}{|c|}{ DIC } & \multicolumn{2}{|c|}{ Total para a população } \\
\hline & $\mathrm{N}$ & $\%$ & $\mathrm{~N}$ & $\%$ & $\mathrm{~N}$ & $\%$ & $\mathrm{~N}$ & $\%$ & $\mathrm{~N}$ & $\%$ & $\mathrm{~N}$ & $\%$ \\
\hline 2002 & 2 & 12,5 & 2 & 25,0 & 2 & 33,3 & 0 & 0 & 0 & 0 & 6 & 16,7 \\
\hline 2004 & 6 & 37,5 & 0 & 0 & 0 & 0 & 1 & 20,0 & 0 & 0 & 7 & 19,4 \\
\hline 2005 & 2 & 12,5 & 2 & 25,0 & 2 & 33,3 & 2 & 40,0 & 0 & 0 & 8 & 22,2 \\
\hline 2007 & 1 & 6,25 & 0 & 0 & 1 & 16,7 & 0 & 0 & 0 & 0 & 2 & 5,56 \\
\hline 2008 & 1 & 6,25 & 0 & 0 & 0 & 0 & 0 & 0 & 1 & 100 & 2 & 5,56 \\
\hline 2009 & 0 & 0 & 0 & 0 & 0 & 0 & 2 & 40,0 & 0 & 0 & 2 & 5,56 \\
\hline Total para a categoria & 16 & 100 & 8 & 100 & 6 & 100 & 5 & 100 & 1 & 100 & 36 & 100 \\
\hline
\end{tabular}

Teste Qui-quadrado ( $X^{2}=46,9 ; p=0,01$; Graus de liberdade=28)

Legenda: CEFAC = Revista Cefac Atualização Científica em Fonoaudiologia; Pro-Fono = Pro-Fono Revista de Atualização Científica; SBFa = Revista da Sociedade Brasileira de Fonoaudiologia; DIC = Revista Distúrbios da Comunicação

cas. O teste Qui-quadrado mostra que a preponderância da vertente positivista, em número absoluto, não tem significância estatística.

Tabela 2. Produção científica por área de atuação fonoaudiológica

\begin{tabular}{lc}
\hline Área & N \\
\hline Linguagem & 17 \\
Motricidade orofacial & 14 \\
Saúde coletiva & 2 \\
Audiologia & 3 \\
\hline Total & 36 \\
\hline
\end{tabular}

Tabela 3. Produção das áreas de atuação por vertente epistemológica

\begin{tabular}{lcccc}
\hline Área & \multicolumn{2}{c}{ Positivista } & \multicolumn{2}{c}{ Dialético-histórica } \\
\cline { 2 - 5 } & $\mathrm{N}$ & $\%$ & $\mathrm{~N}$ & $\%$ \\
\hline Linguagem & 14 & 42,4 & 3 & 100 \\
Motricidade orofacial & 14 & 42,4 & 0 & 0 \\
Saúde coletiva & 2 & 6,06 & 0 & 0 \\
Audiologia & 3 & 9,09 & 0 & 0 \\
\hline Total para a categoria & 33 & 100 & 3 & 100 \\
\hline
\end{tabular}

Teste Qui-quadrado $\left(X^{2}=3,66 ; p=0,30\right.$; Graus de liberdade=3)

A Tabela 4 mostra a distribuição do tipo de procedimento metodológico empregado pelas áreas de atuação.

Quanto à faixa etária, foram encontrados cinco tipos de agrupamentos: crianças; adolescentes; adultos; crianças e adolescentes e todas as faixas etárias. A Figura 1 mostra a distribuição da produção pelo agrupamento etário.

\section{DISCUSSÃO}

Quanto ao crescimento da produção, o período mais produtivo ocorreu entre 2002 e 2005, com decréscimo acentuado entre 2006 e 2008 (Tabela 1). Tais dados demonstram que o crescimento da produção científica fonoaudiológica brasileira sobre PC não acompanha a trajetória identificada em outras áreas do campo fonoaudiológico nacional. Estas apontam haver do início da década de 90 em diante, um aumento significativo nas produções ${ }^{(2,4,5,7,10)}$.

A distribuição da produção pelas áreas de atuação fonoaudiológica espelha o fato de a Motricidade Orofacial e a Linguagem serem as áreas de atuação tradicionalmente ligadas ao trabalho com PC (Tabela 2). Assim, as publicações tratam, preferencialmente, de aspectos ligados à deglutição, à linguagem e a seus processos de avaliação. O pequeno número de trabalhos encontrados em Audiologia, em Saúde Coletiva e a sua ausência na área da Voz, revelam lacunas na pesquisa sobre $\mathrm{PC}$, em vista do fato dessa patologia poder vir acompanhada de problemas de audição e voz e de ser ela uma questão também para a saúde pública.

Quanto à vertente epistemológica, o positivismo subsidiou a maioria dos artigos (Tabela 3), o que significa que o assunto é preferencialmente estudado do ponto de vista do déficit orgânico, centrado no sintoma e na patologia, objetivado pela

Tabela 4. Tipos de procedimento metodológico empregado por área de atuação fonoaudiológica

\begin{tabular}{|c|c|c|c|c|c|c|c|c|c|}
\hline \multirow[t]{2}{*}{ Procedimento metodológico } & \multicolumn{2}{|c|}{ Linguagem } & \multicolumn{2}{|c|}{ Motricidade orofacial } & \multicolumn{2}{|c|}{ Saúde coletiva } & \multicolumn{2}{|c|}{ Audiologia } & \multirow{2}{*}{$\begin{array}{c}\begin{array}{c}\text { Total para a } \\
\text { população }\end{array} \\
\mathrm{N}\end{array}$} \\
\hline & $\mathrm{N}$ & $\%$ & $\mathrm{~N}$ & $\%$ & $\mathrm{~N}$ & $\%$ & $\mathrm{~N}$ & $\%$ & \\
\hline Levantamento & 3 & 17,8 & 12 & 85,7 & 2 & 100 & 1 & 33,3 & 18 \\
\hline Estudo de caso & 11 & 64,7 & 1 & 7,14 & 0 & 0 & 2 & 66,7 & 14 \\
\hline Experimento & 3 & 17,7 & 1 & 7,14 & 0 & 0 & 0 & 0 & 4 \\
\hline Total para a categoria & 17 & 100 & 14 & 100 & 2 & 100 & 3 & 100 & 36 \\
\hline
\end{tabular}

Teste Qui-quadrado $\left(X^{2}=17,6 ; p=0,01 ;\right.$ Graus de liberdade $\left.=6\right)$ 
Faixa etária

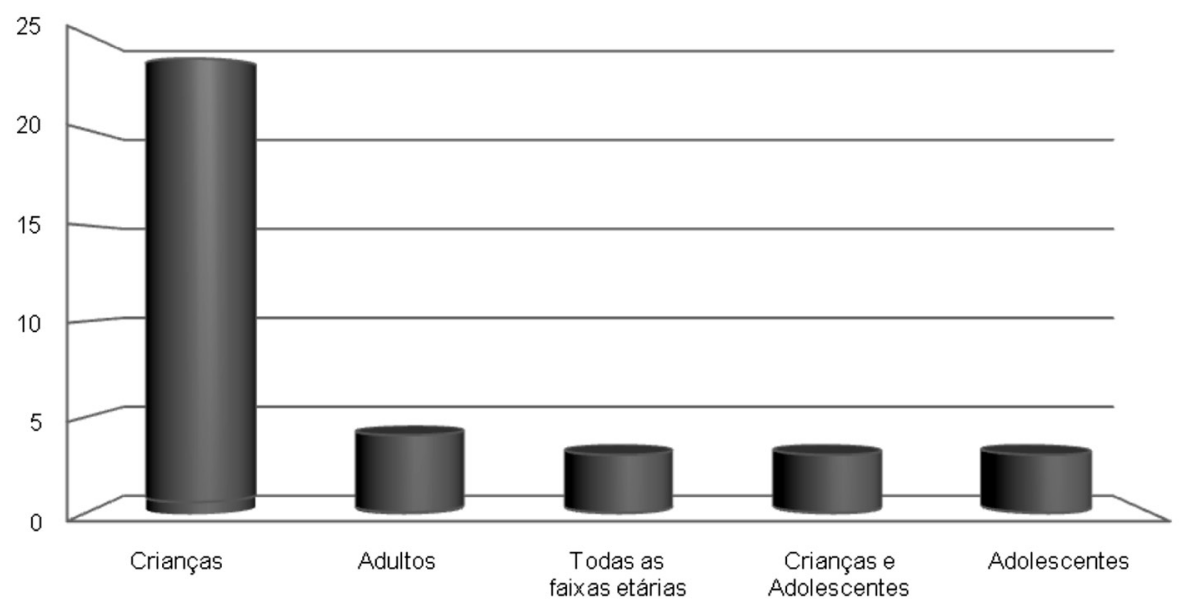

\begin{tabular}{|l|c|c|c|c|c|}
\cline { 2 - 6 } \multicolumn{1}{c|}{} & Crianças & Adultos & $\begin{array}{c}\text { Todas as faixas } \\
\text { etárias }\end{array}$ & $\begin{array}{c}\text { Crianças e } \\
\text { Adolescentes }\end{array}$ & Adolescentes \\
\hline — Número de artigos & 23 & 4 & 3 & 3 & 3 \\
\hline - Porcentagem & 0,64 & 0.11 & 0,08 & 0,08 & 0,08 \\
\hline
\end{tabular}

Figura 1. Produção científica sobre paralisia cerebral, no período de 2002 a 2009

possibilidade de mensuração ${ }^{(8,16)}$.

A vertente dialético-histórica, com três dos 36 trabalhos, também esteve presente na produção estudada, o que está de acordo com outros estudos sobre a produção científica fonoaudiológica brasileira, nos quais também se verificou a presença das vertentes positivista e dialético histórica ${ }^{(7)}$. Todos os artigos que se alinharam à vertente dialético-histórico abordam a área da Linguagem, o que mostra que essa área congrega antagonismos epistemológicos. Mostra também que a construção do conhecimento em Fonoaudiologia não é dominada por uma única tendência de construção do conhecimento e, portanto, que a área não comunga com uma única concepção de homem, de mundo e de linguagem. Isso parece evidenciar a complexidade do objeto da Fonoaudiologia ${ }^{(16)}$.

A distribuição do tipo de procedimento metodológico pelas áreas de atuação fonoaudiológica, (Tabela 4) mostra que em Motricidade Orofacial predominam os levantamentos: estudos com grandes populações ${ }^{(12)}$, com utilização de recursos tecnológicos tais como a videofluoroscopia, o que possibilitou o estudo acurado de muitos distúrbios que acompanham a paralisia cerebral. Estes descrevem aspectos da função oral ou problemas a ela relacionados. Já na área de Linguagem predominaram os estudos de caso, cuja marca é o aprofundamento da pesquisa com poucos indivíduos ${ }^{(12)} \mathrm{com}$ ênfase em aspectos da prática clínica.

Quanto à distribuição da produção pelo agrupamento etário dos sujeitos, (Figura 1), outros estudos também mostram que crianças constituem a faixa etária privilegiada na produção estudada $^{(4,5)}$. Considerando, entretanto, que alterações relacionadas ao envelhecimento tais como alterações de memória, também podem acometer os paralisados cerebrais à medida que envelhecem, trazendo impactos variáveis a seu organismo e exigindo um trabalho terapêutico específico, a faixa etária adulta pode apresentar algum interesse para a pesquisa futura.

\section{CONCLUSÃO}

O levantamento e a caracterização da produção científica fonoaudiológica brasileira sobre PC, no período que vai do ano de 2002 ao primeiro semestre do ano de 2009, mostrou que seu crescimento não acompanha o de outras áreas fonoaudiológicas investigadas, as quais apresentaram marcado crescimento a partir da década de 90 . Mostrou, porém, que sua distribuição pelas vertentes epistemológicas sim acompanha os outros estudos sobre a produção, ao trazer trabalhos tanto na vertente positivista como na vertente dialético histórica, com predominância da primeira.

Com relação aos rumos da pesquisa futura podem-se destacar três aspectos: em relação ao procedimento metodológico, a ausência de estudos de natureza documental; em relação às áreas de atuação fonoaudiológica, a pequena produção em Saúde Coletiva e em Audiologia se comparada à de Linguagem e Motricidade Orofacial, bem como a ausência de estudos na área de Voz; em relação à faixa etária, a escassez de estudos sobre adolescentes e adultos se comparados com o número de estudos envolvendo crianças. 


\begin{abstract}
Purpose: To analyze the Brazilian Speech-Language Pathology scientific production regarding cerebral palsy published between the years of 2002 and 2009 in Speech-Language Pathology journals and in journals from related areas. Methods: Documental descriptive study guided by the selection and analysis of scientific papers about cerebral palsy, regarding: publication period; frequency distribution per period; methodological procedure used; epistemological filiations; age range of the studied subjects; Speech-Language Pathology area of study. Results: Thirty-six articles were found: 28 in Speech-Language Pathology journals, and eight in journals from related areas of study. The publications were mainly concentrated between 2002 and 2005. Most of them regarded the areas of language and orofacial mycology, and the positivist epistemological filiation. The most frequent methodological procedures were data collection and case study. Childhood was the most studied age. Conclusion: The growth of the Brazilian Speech-Language Pathology scientific production regarding cerebral palsy does not follow the growth found in other areas of this science; however, the main epistemological filiation does. Data showed studies about hearing, public health and voice, as well as documental studies and studies regarding adolescents and adults.
\end{abstract}

Keywords: Cerebral palsy; Scientific publication indicators; Scientific and technical publications; Bibliography as topic; Review

\section{REFERÊNCIAS}

1. Freire RM. Uma análise da produção de conhecimento no interior do PEPG em fonoaudiologia. Distúrb Comun. 2005;17(1):37-43.

2. Campanatti-Ostiz H, Andrade CRF. Periódicos nacionais em fonoaudiologia: caracterização de indicador de impacto. Pró-Fono. 2006;18(1):99-110.

3. Tenopir C, King DW. A importância dos periódicos para o trabalho científico. Rev Biblioteconomia Brasília. 2001;25(1):15-26.

4. Berberian AP, Ferreira LP, Corteletti LCBJ, Azevedo JBM, Marques JM. A produção do conhecimento em Distúrbios da Comunicação: análise de periódicos (2000-2005). Rev Soc Bras Fonoaudiol. 2009;14(2):153-9.

5. Berberian AP, Krüger S, Guarinello AC, Massi GAA. A produção do conhecimento em fonoaudiologia em comunicação suplementar e/ou alternativa: análise de periódicos. Rev CEFAC. 2009;11(Supl 2):258-66.

6. Yamamoto $\mathrm{OH}$, Souza CC, Yamamoto ME. A produção científica na psicologia: uma análise dos periódicos brasileiros no período 19901997. Psicol Reflex Crit. 1999;12(2):549-65.

7. Munhoz CMA, Massi G, Berberian AP, Giroto CRM, Guarinello AC. Análise da produção científica nacional fonoaudiológica acerca da linguagem escrita. Pró-Fono. 2007;19(3):249-58

8. Galvão VS, Branco AC. Fonoaudiologia: Epistemologia: implicações pedagógicas e educacionais. Ciênc Educ. 2005;11(2):235-51.

9. Guimarães EL, Tudella E. Reflexos primitivos e reações posturais como sinais indicativos de alterações neurossensoriomotoras em bebês de risco. Pediatria (São Paulo). 2003;25(1/2):28-34
10. Campanatti-Ostiz H, Andrade CRF. Periódicos nacionais em fonoaudiologia: caracterização estrutural. Rev Soc Bras de Fonoaudiol. 2005;10(3):147-54.

11. Russo ICP, Ferreira LP. Fonoaudiólogos doutores no Brasil: análise das teses segundo áreas de atuação e programas. Pró-Fono. 2004;16(1):11930.

12. Gil AC. Métodos e técnicas em pesquisa social. São Paulo: Atlas; 2000. $207 \mathrm{p}$.

13. Brasil. Conselho Federal de Fonoaudiologia [Internet]. Resolução CFFa $\mathrm{n}^{\mathrm{o}}$ 320, de 17 de fevereiro de 2006. Dispõe sobre as especialidades reconhecidas pelo Conselho Federal de Fonoaudiologia, e dá outras providências. [citado 2009 out 25]. Disponível em: http://www. fonoaudiologia.org.br/\#

14. Spink MJP. Caminando sobre huevos: una reflexión construccionista sobre la investigación. Athenea Digital. 2006;9:168-83.

15. Oliveira GJ, Oliveira ES, Leles CR. Tipos de delineamento de pesquisa de estudos publicados em periódicos odontológicos brasileiros. Rev Odonto Ciênc. 2007; 22(55):42-7.

16. Oliveira PS, Friedmann S. A clínica da gagueira: diferentes paradigmas e suas consequências. In: Silva OS, David RHF, organizadores. Cadernos da fonoaudiálogo: linguagem. São Paulo: Lovise; 2006. Vol. 1, p. 7-13. 